\title{
Development of Metal-resin Composite Restorative Material Part 5: Evaluation of the Bonding between Ag-Sn Particle and 4-META Coupling Agent of the Metal-resin Composite
}

\author{
Somchai URAPEPON, Kiyoshi KAKUTA ${ }^{1}$ and Hideo OGURA ${ }^{1}$ \\ Prosthodontics Department \\ Faculty of Dentistry, Mahidol University \\ 6, Yothi Road, Rachathevi, Bangkok, 10400, Thailand \\ ${ }^{1}$ Department of Dental Materials Science, \\ School of Dentistry at Niigata, The Nippon Dental University \\ 1-8 Hamaura-cho, Niigata City, Niigata, 951-8580, Japan
}

Received December 16, 2002/Accepted March 28, 2003

\begin{abstract}
This study evaluated the oxide characteristics on a Ag-Sn particle surface modified by acid and heat in order to understand the bonding mechanism. Nine different Ag-Sn particles including standard $\mathrm{Sn}, \mathrm{SnO}$ and $\mathrm{SnO}_{2}$ were evaluated by X-ray Photoelectron Spectroscopy (XPS). The particles were also used for preparing the composite to test the flexural properties following the ISO 4049. A high correlation between flexural strength of the composites and Sn3d binding energy of their filler particles was found. Different oxide forms $\left(\mathrm{SnO}\right.$ and $\left.\mathrm{SnO}_{2}\right)$ showed different adhesive abilities to bond with 4-META, regarding the difference in flexural strength of the composite. The highest flexural strength composite showed Sn3d binding energy of its filler at $486.4 \mathrm{eV}$, which was the $\mathrm{SnO}$ binding area. This implies that $\mathrm{SnO}$ was responsible for a good bond between particle surface and 4-META coupling agent.
\end{abstract}

Key words: Silver alloy, Bonding, Dental composite

\section{INTRODUCTION}

The metal-resin composite (MRC) using Ag-Sn alloy particles as filler, 4-META as a coupling agent and UDMA-TEGDMA as resin matrix, have been developed ${ }^{1,2)}$. The mechanical and physical properties of the composite showed a promising good posterior composite ${ }^{1,2)}$. The bonding between filler particles and the coupling agent through the resin matrix is the critical key point for the strength of the composite in this system. Therefore, a good understanding of the bonding mechanism is necessary to improve this composite. It had been hypothesized and reported in several studies $^{3-9)}$ that adhesive resin formed a chemical bond with metal oxide films, especially of base metal ( $\mathrm{In}, \mathrm{Cu}, \mathrm{Zn}$ and $\mathrm{Sn}$ ). Although these oxide films are considered to play an important role in promoting the adhesive ability of adhesive resin to alloy, the detail characteristics of these oxide films and adhesive resins have not been well understood. Ohno et $a l^{3,4)}$ reported that the base metal content and the area fraction of the oxide on the surface had a great effect on the adhesive ability. Urapepon et al. ${ }^{2)}$ reported that an appropriate heat treatment for Ag-Sn particle was required to make a good bond with 4-META resin, however, the responsible oxide 
form could not be differentiated. Since the metal element in the composite filler had fixed at the greatest mechanical and physical properties, the important point to be clarified in this study were the detailed oxide characteristics in high temperature oxidation which play the important role on the adhesive ability to 4-META.

In the previous study ${ }^{2)}$, although the effect of the acid and heat treatment were carried out, detailed information about the acid concentration and bonding mechanism was not well described. Therefore, the objective of this study was to investigate the oxide characteristics on the Ag-Sn particle surface, modified by acid and heat in order to understand the bonding mechanism through the strength of the composite.

\section{MATERIALS AND METHODS}

Ag-Sn spherical alloy particles ( 73.2 mass\% Ag, 26.8 mass\% Sn, Lot no. 9804AS750) were produced by the research unit, Faculty of Dentistry, Mahidol University, Thailand, using the atomization method. Particles bigger than $53 \mu \mathrm{m}$ were crushed by a ball-milling machine (Pulverisette, Fritsch, Germany) at speed 8 on the machine's dial pad $(595 \mathrm{rpm})$ for $5 \mathrm{hr}$ to produce irregular particles. After crushing, the particles were then sieved with $20 \mu \mathrm{m}$ mesh. The particles less than $20 \mu \mathrm{m}$ were utilized for the filler of MRC.

\section{Alloy particle surface treatment}

The alloy particles were immersed in either 2 or $4 \% \mathrm{HCl}$ at $80^{\circ} \mathrm{C}$ and slowly stirred with a magnetic stirrer for $30 \mathrm{~min}$. After the acid treatment, the particles were thoroughly washed with distilled water until no residual acid remained on the surface, verified using $\mathrm{AgNO}_{3}$. The particles were filtered and dried at $40^{\circ} \mathrm{C}$ for $1 \mathrm{hr}$. In addition to these treatments, water washing in the same conditions as the acid treatment was included.

After drying, the particles were divided into three groups. One of the three groups was used as it was, without any heat treatment. The other two groups were placed in an oven for heat treatment at either $150^{\circ} \mathrm{C}$ or $300^{\circ} \mathrm{C}$ for $5 \mathrm{~min}$. Therefore, 9 different particles treated with different acid concentrations and heating temperatures were prepared.

The composite specimen preparation and flexural test

The preparation of the MRC specimen and the flexural test were carried out following the procedure reported in the previous study ${ }^{10)}$. The particles were coupled with 10 mass\% 4-META-acetone solution containing 1 mass \% BPO for $30 \mathrm{sec}$ and dried. The coupled particles were mixed with monomer (UDMA, 75 mass \% and TEGDMA, 25 mass \%) containing 0.25 mass\% DMPT to prepare the composite. The filler content was $93 \%$ by weight. The flexural specimens were prepared and tested following the ISO 4049: 1998 for resin-based filling materials. Three replications were prepared for each of the nine conditions. The data of flexural strength and flexural modulus 
were analyzed using two-way analysis of variance to observe the effect of two factors, which were the acid concentration and the heating temperature. The equation was computed from the orthogonal polynomials, which were calculated using the significant terms of the factors obtained from the results of ANOVA. The iso-value curves for both flexural strength and flexural modulus were drawn using the above mentioned equation.

\section{Particle surface analysis by X-ray photoelectron spectroscope (XPS)}

Nine different particles treated with different acid concentrations and heating temperatures, including standard samples of $\mathrm{Sn}, \mathrm{SnO}$ and $\mathrm{SnO}_{2}$, were dried in a desiccator under a vacuum at room temperature for $12 \mathrm{hr}$ and then placed on carbon tape. The surface of the particles was analyzed using an X-ray photoelectron spectroscope (JPS-9000MC, JEOL Co. Ltd., Tokyo, Japan) with Al K $\alpha$ radiation under a pressure of $2 \times 10^{-5} \mathrm{~Pa}$. The binding energy of the measured photoelectron peak was calibrated by the $\mathrm{C} 1 \mathrm{~s}$ peak at a binding energy of $284.6 \mathrm{eV}$.

\section{RESULTS}

\section{Flexural strength and flexural modulus of the composite}

Table 1 shows the summary of the flexural strength and flexural modulus of the experimental composite. The flexural strength ranged from 85.4 to $102.4 \mathrm{MPa}$, and the flexural modulus from 9.1 to $11.8 \mathrm{GPa}$. Table 2 shows the results of ANOVA for the

Table 1 Summary of the flexural strength, flexural modulus of the experimental composite and Sn3d binding energy of the filler particle surface including $\mathrm{Sn}, \mathrm{SnO}$ and $\mathrm{SnO}_{2}$ standard samples

\begin{tabular}{|c|c|c|c|c|}
\hline \multicolumn{2}{|c|}{ Metal-resin composite } & \multirow{2}{*}{$\begin{array}{l}\text { Flexural } \\
\text { strength: } \\
\mathrm{MPa} \text { (SD) }\end{array}$} & \multirow{2}{*}{$\begin{array}{l}\text { Flexural } \\
\text { modulus: } \\
\text { GPa }(\mathrm{SD})\end{array}$} & \multirow{2}{*}{$\begin{array}{c}\text { Sn3d binding } \\
\text { energy: } \\
\text { eV }\end{array}$} \\
\hline Acid conc. $(\%)$ & Heating temp. $\left({ }^{\circ} \mathrm{C}\right)$ & & & \\
\hline 0 (water) & Room temp & $85.5(5.2)$ & $9.1(0.9)$ & 486.8 \\
\hline 0 (water) & 150 & $88.9(1.8)$ & $9.3(0.5)$ & 486.7 \\
\hline 0 (water) & 300 & $85.4(0.9)$ & $9.2(0.5)$ & 486.8 \\
\hline 2 & Room temp & $93.9(1.7)$ & $10.6(0.6)$ & 486.2 \\
\hline 2 & 150 & $102.4(3.5)$ & $10.3(0.4)$ & 486.4 \\
\hline 2 & 300 & $85.8(3.8)$ & $10.8(0.7)$ & 486.8 \\
\hline 4 & Room temp & $93.2(4.2)$ & $10.2(0.6)$ & 486.1 \\
\hline 4 & 150 & $99.8(4.7)$ & $11.8(1.1)$ & 486.2 \\
\hline 4 & 300 & $89.8(4.2)$ & $10.4(1.6)$ & 486.6 \\
\hline \multicolumn{2}{|c|}{ As crushing } & & & 486.3 \\
\hline \multicolumn{2}{|r|}{$\mathrm{Sn}$} & & & 484.9 \\
\hline \multicolumn{2}{|r|}{$\mathrm{SnO}$} & & & 486.5 \\
\hline \multicolumn{2}{|r|}{$\mathrm{SnO}_{2}$} & & & 486.7 \\
\hline
\end{tabular}


Table 2 Results of ANOVA for the flexural strength and flexural modulus of the composite

\begin{tabular}{lrrrrc}
\hline \multicolumn{1}{c}{ Source } & \multicolumn{1}{c}{ SS } & df & MS & F & P \\
\hline Flexural strength: & & & & & \\
A: Acid Conc. & 343.73 & 2 & 171.87 & 13.16 & $<0.01^{* *}$ \\
B: Heating T. & 464.31 & 2 & 232.15 & 17.78 & $<0.01^{* *}$ \\
AxB & 130.16 & 4 & 32.54 & 2.49 & 0.08 \\
Error & 235.05 & 18 & 13.06 & & \\
Total & 1173.25 & 26 & & & \\
\hline Flexural modulus: & & & & & \\
A: Acid Conc. & 12.47 & 2 & 6.24 & 8.96 & $<0.01^{* *}$ \\
B: Heating T. & 1.48 & 2 & 0.74 & 1.06 & 0.37 \\
AxB & 3.75 & 4 & 0.94 & 1.35 & 0.29 \\
Error & 12.53 & 18 & 0.70 & & \\
Total & 30.23 & 26 & & & \\
\hline
\end{tabular}

** indicate highly significant at $99 \%$

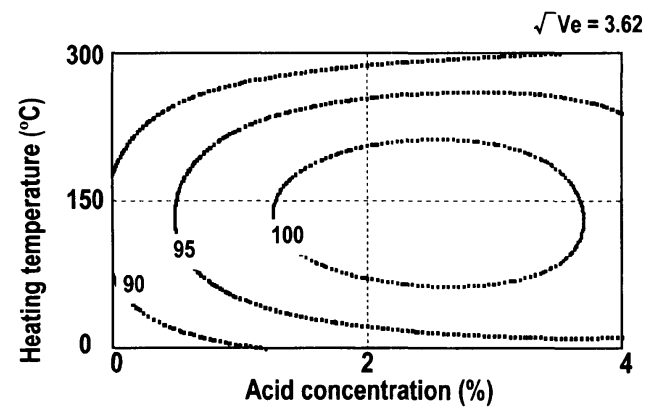

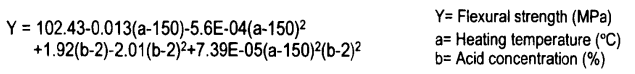

Fig. 1 The flexural strength of the experimental composite.

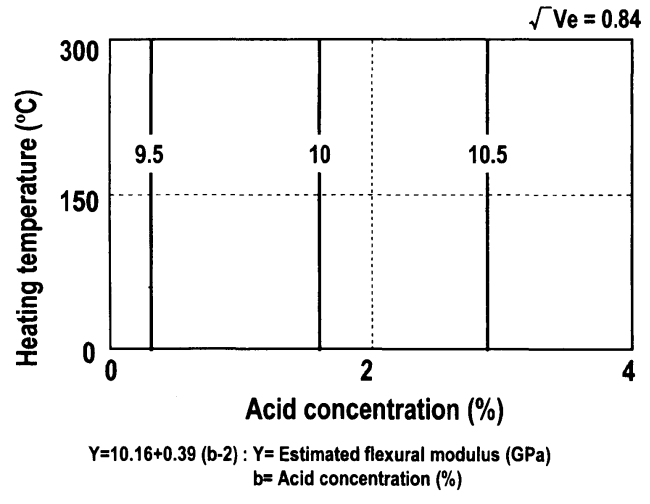

Fig. 2 The flexural modulus of the experimental composite.

flexural strength and flexural modulus of the composite. The flexural strength was significantly influenced by both main factors without their interaction $(p<0.01)$. The flexural modulus was significantly influenced only by acid concentration $(p<0.01)$. Figs. 1 and 2 show the flexural strength and the flexural modulus of the experimental composite, which were drawn by the equation shown below the figure. The square root $\mathrm{Ve}$ seen in the figure is the square root of the mean square of error obtained from ANOVA. As shown in Fig. 1, the flexural strength increased either as the acid concentration increased (up to 2.5-3.0\%) or as the heating temperature increased (up to $\left.150^{\circ} \mathrm{C}\right)$. The highest flexural strength of the composite in this study $(102.4 \mathrm{MPa})$ was obtained from the particle with $2 \%$ acid and $150^{\circ} \mathrm{C}$ heat treatment. Fig. 2 shows that the flexural modulus increased when the acid concentration increased. However, when the orthogonal polynomials were calculated using the significant terms of 


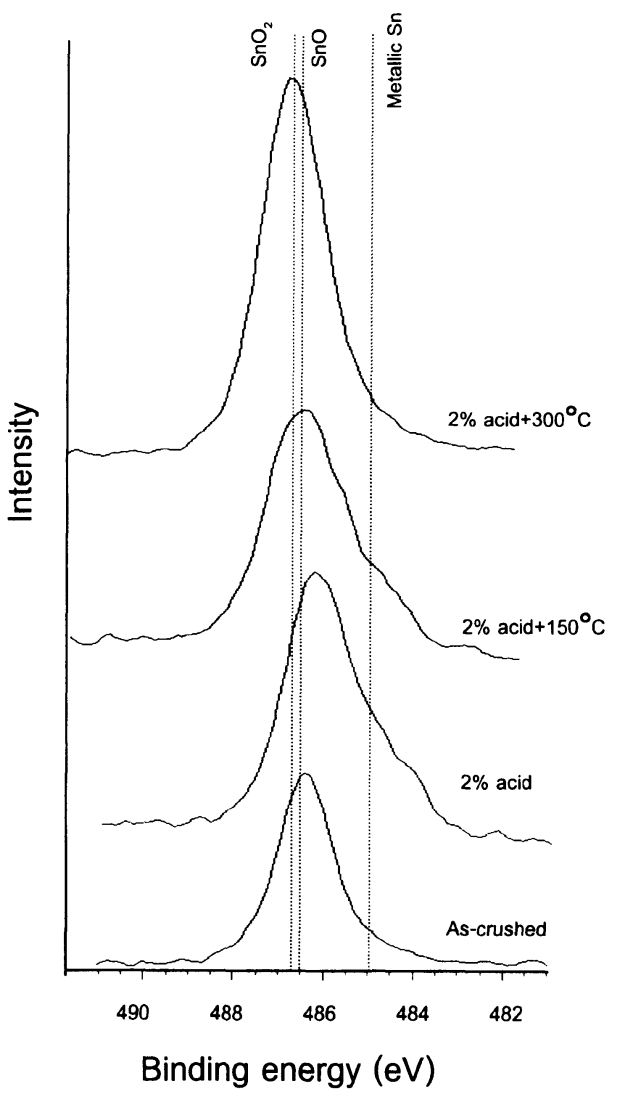

Fig. 3 The Sn3d spectra obtained from as-crushed, $2 \%$ acid-treated, $2 \%$ acid- and $1500^{\circ} \mathrm{C}$ heat-treated and $2 \%$ acid- and $300{ }^{\circ} \mathrm{C}$ heat-treated particles.

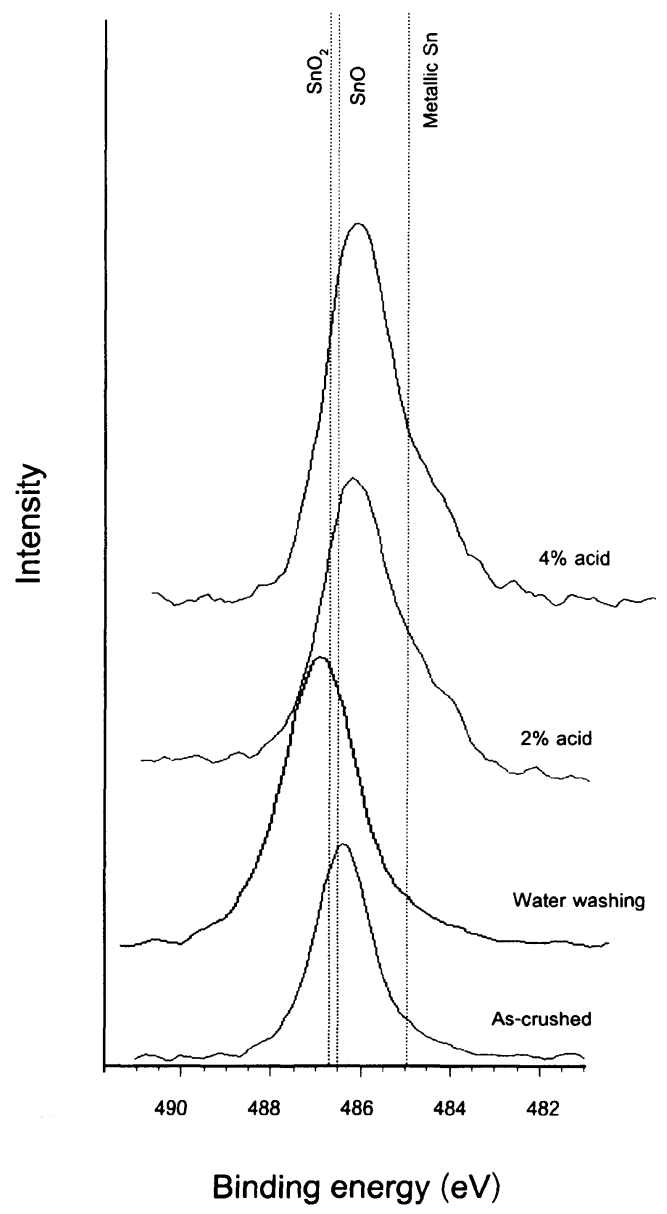

Fig. 4 The Sn3d spectra obtained from the as-crushed, water washing, $2 \%$ acidtreated and $4 \%$ acid-treated particles.

factors, a significant difference in the second level (between $2 \%$ and $4 \%$ acid concentration) was not found.

\section{Oxide analysis on particle surface}

The Sn3d binding energy of the filler particle surface including as-crushed particles as well as $\mathrm{Sn}, \mathrm{SnO}$ and $\mathrm{SnO}_{2}$ standard samples were shown in Table 1. Fig. 3 shows the Sn3d spectra obtained from as-crushed, $2 \%$ acid-treated, $2 \%$ acid- and $150^{\circ} \mathrm{C}$ heattreated and $2 \%$ acid- and $300^{\circ} \mathrm{C}$ heat-treated particles. The spectra had a wide half width due to the mixture of metallic $\mathrm{Sn}$ and their oxidation states as $\mathrm{SnO}$ and $\mathrm{SnO}_{2}$. The spectrum shifted to a higher oxidation state as the temperature increased. Fig. 4 shows the Sn3d spectra obtained from the as-crushed, water washing, $2 \%$ acid- 


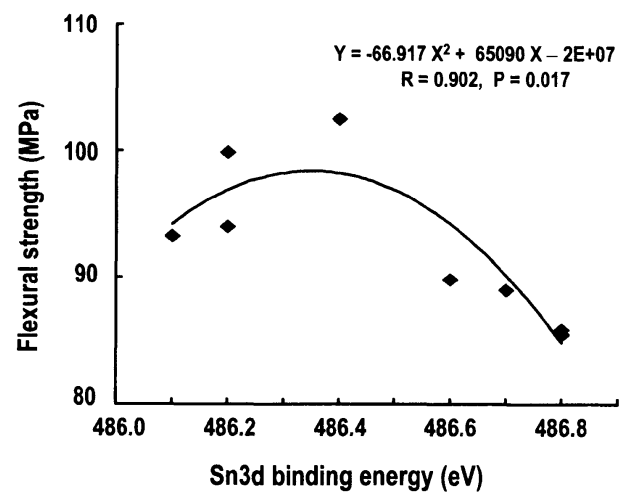

Fig. 5 The correlation between the flexural strength of the composite and its filler surface Sn3d binding energy.

treated and $4 \%$ acid-treated particle. The spectrum shifted to a higher oxidation state when the particle was washed in water and to lower oxidation state or more metallic Sn when it received acid treatment. The spectrum does not clearly show the different between $2 \%$ and $4 \%$ acid treatment.

A correlation between the flexural strength of the composite and its filler surface Sn3d binding energy was found $(\mathrm{R}=0.902, \mathrm{P}=0.017)$ and shows on Fig. 5 . The composite showed the highest strength when its filler has Sn3d binding energy around $486.4 \mathrm{eV}$, which mean the majority of its filler surface was composed of $\mathrm{SnO}$.

\section{DISCUSSION}

In this study, the Ag3d binding energy of the filler particle surface, including $\mathrm{Ag}$ and $\mathrm{AgO}_{2}$ standard samples was also evaluated. However, there was no relation or meaning to interpret. Therefore, the data of the $\mathrm{Ag} 3 \mathrm{~d}$ were not reported.

In this study, acid and heat treatment were selected to modify the surface for analyzing the surface product that was responsible for the bonding because of the expectation that contamination would be removed and oxide would be formed when a particle was acid- and heat-treated, respectively. The results in this study showed that the acid and heat treatment affected the flexural strength of the composite, while only acid treatment affected the flexural modulus. The past studies ${ }^{2,10,11)}$ clearly showed that a wider groove at the grain boundary was found, and became much wider or had pitting on the grain surface with higher acid concentration. The wider grooves increase the bonding area and mechanical interlocking between the alloy particle and resin matrix. Increasing the bonding area will decrease the matrix mass between filler, regarding increasing the flexural modulus. This might be the reason why acid treatment affected both flexural strength and flexural modulus. These results were conformed in vice versa in Kakuta et al.'s study ${ }^{12)}$. They studied the acid 
and heat treatment on $\mathrm{Ag}$-In, which require a higher concentration to widen the groove of the grain boundary. Therefore, they did not found any effect of acid treatment on both flexural strength and flexural modulus, only a trend to be higher with acid concentration.

As mentioned above, we expected the oxide formation on the surface when the particle got the heat treatment. The results in this study suggested that there was a significant difference in the flexural strength of the composite according to the temperature of heat treatment. This meant that the condition of the oxide, which was formed by heat, affected the flexural strength of the composite. Fig. 1 shows that the flexural strength increased up to $150^{\circ} \mathrm{C}$ then decreased when the heating temperature increased. In this study, the highest flexural strength was obtained at condition $2 \%$ acid and $150^{\circ} \mathrm{C}$ heat treatment. Several studies ${ }^{3-9)}$ found that a different metal oxide, thickness of the oxide layer or a different metal oxide form affected the bonding strength. These findings imply that the proper oxide layer or oxide form might be the main key to the bonding between particle surfaces and the coupling agent in regard to flexural strength of the composite. However, the specific metal oxide form cannot be distinguished.

From the particle surface analysis by XPS in Fig. 4, the Sn3d spectra shifted to more metallic-tin when acid treatment was received. This fulfills our expectation that acid treatment could remove any contaminated oxide on the surface. Unexpected data was found on water washing particles; the spectra shifted to a higher oxidation state $\left(\mathrm{SnO}_{2}\right)$, which was different from the spectra of as-crushed particles. Ohno et $a l^{4)}$ have reported that $\mathrm{H}_{2} \mathrm{O}$ molecules on the alloy surface exhibit good adhesion to 4-META and alloy surfaces. This may be the reason why the flexural strength of the water washed composite was different from the as-crushed composite in the past study ${ }^{11)}$.

Fig. 3 shows the $\mathrm{Sn} 3 \mathrm{~d}$ spectra shifted to an oxidation state $(\mathrm{SnO})$ when heat treatment was received at $150^{\circ} \mathrm{C}$, and shifted to a higher oxidation state $\left(\mathrm{SnO}_{2}\right)$. This suggested that the oxide was formed on the particle surface by heat treatment and different oxide formed with the rising temperature. These different oxide forms were expected to give different bond strengths to the coupling agent. As shown in Fig. 5, a high correlation $(\mathrm{R}=0.902, \mathrm{P}=0.017)$ between the flexural strength of the composite and its filler surface Sn3d binding energy $(\mathrm{eV})$ was found. A correlation was found in a convex parabola shape. This confirmed the hypothesis that 4-META has different adhesion ability according to different oxide types and forms. The highest strength of the composite was shown at binding energy around $486.4 \mathrm{eV}$, which was $\mathrm{SnO}$ binding energy area. This result implies that $\mathrm{SnO}$ is the most responsible for the adhesive ability between 4-META and alloy surface.

\section{CONCLUSION}

Both acid and heat treatment affected the flexural strength, while only acid treatment affected the flexural modulus of the composite. The highest flexural strength 
of the composite $(102.4 \mathrm{MPa})$ was obtained with $2 \% \mathrm{HCl}$ and $150^{\circ} \mathrm{C}$ heat treatment. The flexural moduli ranged from 9.1-11.8 GPa.

When acid treatment was done, the XPS result showed more metallic-tin (less oxide) on the surface, while heat treatment showed more oxide forming on the surface. A higher oxidation state oxide form $\left(\mathrm{SnO}_{2}\right)$ was formed when the heating temperature increased. A different oxide form ( $\mathrm{SnO}$ and $\mathrm{SnO}_{2}$ ) showed different adhesive ability to 4-META regarding the difference in the strength of the composite. A high correlation between flexural strength of the composite and Sn3d binding energy of their filler particles was found $(R=0.902, P=0.017)$. The filler surface of the highest flexural strength composite showed Sn3d binding energy at $486.4 \mathrm{eV}$, which was the $\mathrm{SnO}$ binding area. This implies that $\mathrm{SnO}$ was responsible for a good bond between the particle surface and the 4-META coupling agent, regarding the high flexural strength of the composite.

\section{ACKNOWLEDGMENTS}

The authors thank Professor Yukio Miyagawa for providing the iso-curve drawing program, and valuable statistical consultation. X-ray photoelectron spectroscopy was performed at Advance Research Center, School of Dentistry at Niigata, The Nippon Dental University, Japan.

This work was partially supported by Thailand Research Fund (PDF/34/2543).

\section{REFERENCES}

1) Kakuta, K., Urapepon, S., Miyagawa, Y., Ogura, H., Suchatlampong, C. and Rittapai, A.: Development of metal-resin composite restorative material; Experimental composite containing silver-tin alloy as filler and 4-META as coupling agent, Dent Mater $J$ 18(1): $1-10,1999$.

2) Urapepon, S., Kakuta, K., Miyagawa, Y., Ogura, H., Suchatlampong, C. and Rittapai, A.: Development of metal-resin composite restorative material; Part 2 Effects of acid and heat treatment of silver-tin filler particles on the flexural properties of the metal-resin composite, Dent Mater J 18(2) : 144-154, 1999.

3) Ohno, H., Yamane, Y., Endo, K., Araki, Y. and Iizuka, Y.: Adhesion of adhesive resin to dental precious metal alloys; Part 1, Dent Mater $J$ 17(4):275-284, 1998.

4) Ohno, H., Endo, K., Yamane, Y. and Kawashima, I.: Adhesion of adhesive resin to dental precious metal alloys; Part 2, Dent Mater J 17(4) : 285-293, 1998.

5) Salonga, J. P., Matsumura, H., Yasuda, K. and Yamabe, Y.: Bond strength of adhesive resin to three nickel-chromium alloys with varying chromium content, $J$ Prosth Dent $72: 582-584,1994$.

6) Ohno, H., Endo, K., Yamane, Y. and Kawashima, I.: XPS study on the weakest zone in the adhesion structure between resin containing 4-META and precious metal alloys treated with different surface modification methods, Dent Mater $J$ 20(1):90-102, 2001.

7) Yamane, Y., Ohno, H. and Endo, K.: Mechanism of adhesion between 4-META resin and alloys based on Bolger's acid-base interaction, Dent Mater $J$ 20(1) : 63-74, 2001.

8) Eder, A. and Wickens, J.: Surface treatment of gold alloys for resin adhesion, Quintessence Int 27(1) : 35-40, 1996.

9) Peachey, A., Eder, A., Ibbetson, R. and Pearson, G.: Surface treatment of alloys for resin adhesion: an in-vitro study, Clin Mater 17(2) : 105-109, 1994. 
10) Urapepon, S. and Ogura, H.: Metal-resin composite restorative material using powderliquid system, Dent Mater $J$ 18(3) : 278-294, 1999.

11) Urapepon, S., Kakuta, K., Ogura, H., Suchatlampong, C. and Rittapai, A.: Development of metal-resin composite restorative material. Part 3 Flexural properties and condensability of metal-resin composite using Ag-Sn irregular particles, Dent Mater $J$ 19(2) : 186-195, 2000.

12) Kakuta, K., Urapepon, S., Miyagawa, Y., Ogura, H., Yamanaka, M., Suchatlampong, C. and Rittapai, A.: Development of Metal-resin Composite Restorative Material Part 4 Flexural Strength and Flexural Modulus of Metal-resin Composite using Ag-In alloy Particles as Filler, Dent Mater J 21(2): 181-190, 2002. 